\title{
Amorphous and Cellular Computing
}

Hal Abelson, Gerald Jay Sussman \& Thomas F. Knight, Jr.

Artificial Intelligence Laboratory

Massachusetts Institute of Technology

Cambridge, Massachusetts 02139

http://www.ai.mit.edu

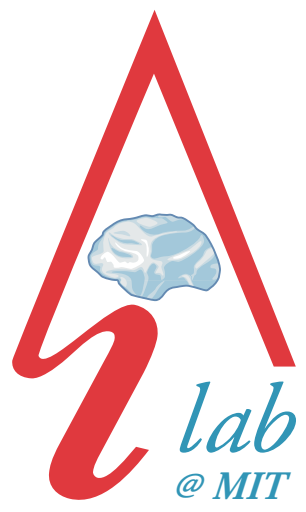

The Problem: The objective of this work is to create a new engineering discipline for reliably obtaining useful, prespecified behavior from vast numbers of asynchronous, irregularly arranged information-processing units that communicate with only a few neighbors.

Progress in microfabrication and in bioengineering will make it possible to assemble such amorphous systems at almost no cost, provided that 1) the units need not all work correctly; 2) the units are identically programmed; and 3) there is no need to manufacture precise geometrical arrangements of the units or precise interconnections among them. Harnessing these systems for information processing and intelligent materials requires augmenting traditional information processing with new perspectives and techniques from biology.

Motivation: Computer science is currently built on a foundation that largely assumes the existence of a perfect infrastructure. Integrated circuits are fabricated in clean-room environments, tested deterministically, and discarded if even a single defect is uncovered. Entire software systems fail with single-line errors. In contrast, biological systems rely on local computation, local communication, and local state, yet they exhibit tremendous resiliance. No existing engineering framework creates such complex structure from simple, potentially faulty components or maintains such complex behavior of existing structures under dramatic environmental change.

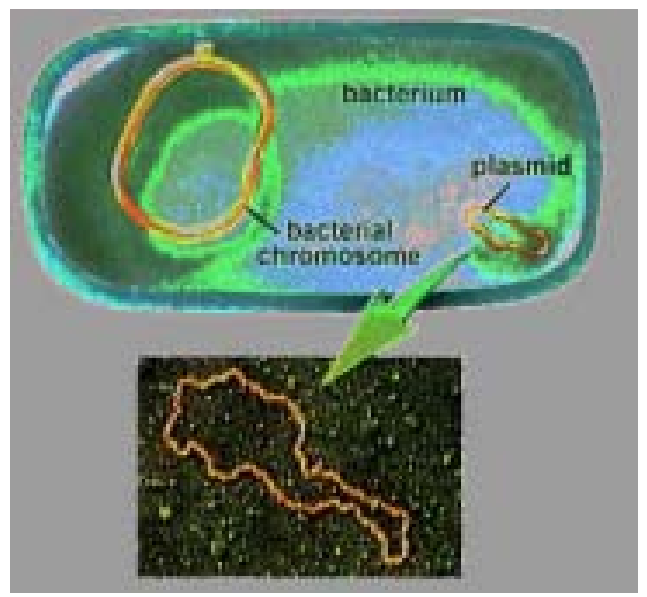

Figure 1: Programming a bacterial cell by creating reconbinant-DNA gates

Previous Work: A major thrust of our work is to make it possible to use living cells as a substrate for engineering, and to program colonies of simple bacterial cells to be test beds for the organizational principles of amorphous computing. We have demonstrated, by simulation of reaction kinetics, the theoretical feasibility of building a family of logic gates where the signals are represented by concentrations of DNA-binding proteins, and where the nonlinear amplification is implemented by in vivo DNA-directed protein synthesis.

We have also installed and equipped a complete recombinant DNA laboratory for constructing and characterizing "cellular logic" gates based on these theoretical foundations. In the lab, we have demonstrated the fundamentals of "genetic process engineering" - taking existing genertic regulatory elements and modifying their DNA encoding so that they can be used in constructing complex $\mathrm{i}_{i} i_{i}$ in vivo ${ }_{i} / i_{i}$ digital-logic circuits. 

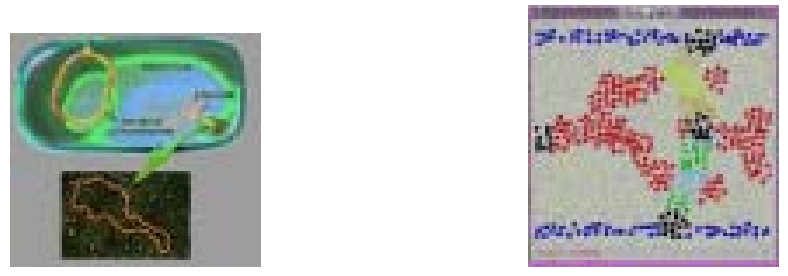

Figure 2: (a) Programming bacterial cells by creating reconbinant-DNA gates. (b) Programming an amorphous medium to form complex patterns (in this case, the connection graph of a CMOS inverter

Outside the lab, we have developed a prototype simulation model for controlling programmable materials. This work shows how to organize a program to direct a sheet consisting of a vast number of autonomous, asynchronous, identically programmed, locally communicating, and irregularly placed agents, that can individually deform ("cells"), to cooperate to construct a large family of globally-specified predetermined shapes.

Approach: Our general approach is to invent new programming paradigms, languages, and algorithms for controlling amorphous computing agents, as well as to investigate prototypes, both in traditional silicon technology and in molecular biology.

Impact: Progress in amorphous and cellular computing could have a profound impact on computer science, biology, and microelectronics. In biology, we can perhaps address longstanding questions of morphogenesis, and discover a computational understanding of the developmental process. In computer science, we can engineer robust computational infrastructures, out of unreliable components. In microelectronics, we can biochemically pattern nanoscale information rich substrates with atomic precision. We can incorporate robust local computation, sensing, and effectors arrays into structures which exhibit sophisticated global behavior.

The amorphous computing framework will give us the power to take control of some biological processes, and to design and construct biological cells and cell assemblies with prespecified behaviors. Such controlled biological mechanisms will give us the ability to construct of novel materials with engineered nanoscale structures.

Future Work: Over the coming year, we will continue to engineer and characterize a wide variety of mix-andmatch genetic components that can be added to a cell to build the behaviors that we desire. These components will cover intracellular signaling and control mechanisms, sensory mechanisms, effector mechanisms, and intercellular communication mechanisms. We will also continue our work in progammable materials, with languages for directing the self-assembly of pre-specified three-dimensional shapes.

Research Support: Support for this research is provided in part by the Advanced Research Projects Agency of the Department of Defense under Office of Naval Research contract N00014-96-1-1228.

\section{References:}

[1] Hal Abelson, Don Allen, Daniel Coore, Chris Hanson, George Homsy, Thomas F. Knight Jr., Radhika Nagpal, Erik Rauch, Gerald Jay Sussman, and Ron Weiss. Amorphous computing. Comm. of the ACM, May 2000. 